Optical processes in quantum confined semiconductors

This content has been downloaded from IOPscience. Please scroll down to see the full text. 1993 Phys. Scr. 1993470

(http://iopscience.iop.org/1402-4896/1993/T49B/016)

View the table of contents for this issue, or go to the journal homepage for more

Download details:

IP Address: 193.255.193.159

This content was downloaded on $02 / 11 / 2014$ at $14: 17$

Please note that terms and conditions apply. 


\title{
Optical Processes in Quantum Confined Semiconductors
}

\author{
Roberto Cingolani \\ Dipartimento di Scienza dei Materiali, Università di Lecce, Via Arnesano, 73100 Lecce, Italy
}

Received March 30, 1993; accepted April 21, 1993

\begin{abstract}
A short overview of the optical properties of quantum wires and quantum dots is given. The results of recent spectroscopic experiments are discussed to elucidate the correlations between the structural and the optical properties of low-dimensional systems.
\end{abstract}

\section{Introduction}

The quantum size effect in nanometer heterostructures has been exploited in a number of technological applications and for the study of quantum-mechanical processes. Most of the work has been dealing with quantum wells and superlattices in which the carrier motion was confined along one direction (the growth direction) [1]. In the last years increasing efforts have been made to further reduce the heterostructure dimensionality in order to fabricate true one dimensional (1D) quantum wires and zero-dimensional (0D) quantum dots. This field is relatively at an early stage and many technological difficulties in the fabrication of quantum wires and dots as well as in the understanding of their physical properties have still to be solved. Nevertheless, the most attractive possibility to get tunable photonic devices like low-threshold lasers and non-linear modulators based on confined systems with sharp density of states and fast non-linear response are still keeping the attention of the scientific community. In this paper we discuss the optical properties of low dimensional systems, with particular attention to recent experiments in GaAs quantum wires and II-VI quantum dots. Different classes of processes are discussed depending on the density of elementary excitations created in the heterostructure and on the frequency of the exciting electromagnetic field (i.e. optical excitation in the transparency region, in the absorption continuum or in resonance with any real state).

\section{Experimental}

A number of spectroscopic methods can be used to investigate the electronic states and the optical transitions in quantum wires and quantum dots. On a first step the search for confined states can be performed by photoluminescence (PL), photoluminescence-excitation (PLE), photoreflectance (PR) and absorption (ABS) spectroscopy. The obtained data are compared with the calculated quantization energies, which rely on the structural and morphological parameters determined by high-resolution double-crystal X-ray diffraction [2] and electron-microscopy [3]. The non-linear response and the excitonic selection rules of quantum wires can be studied by two-photon-absorption induced photoluminescence-excitation (TPA-PLE) under different polarization geometries [4]. The dense electron-hole plasma regime and the dynamics of the radiative recombination processes are studied by time-resolved high-excitation intensity photoluminescence, using a ps laser and a streak camera. This provides information on the band gap renormalization and on the hot carrier cooling rate. All measurements are carried out in a wide temperature range $(10 \mathrm{~K}-300 \mathrm{~K})$ and by tuning the excitation intensity in the range $0.1 \mathrm{~W} \mathrm{~cm}^{-2}-10 \mathrm{MW} \mathrm{cm}^{-2}$.

Quantum wires of different structure can be studied, namely, rectangular quantum wires prepared by holographic lithography and subsequent plasma etching, or Vshaped quantum wires fabricated by MBE growth on patterned substrates. The rectangular quantum wires are fabricated by processing a multiple $\mathrm{GaAs} / \mathrm{AlGaAs}$ quantum well heterostructure of typical thickness $10 \mathrm{~nm}$, with a lateral width of the order of $50 \mathrm{~nm}$. Details on the structural properties of the wires are reported in Ref. [2]. The Vshaped wires are obtained by the controlled tapering of a bent $\mathrm{GaAs}$ quantum well cladded by two GaAs/AlAs superlattices deposited onto a wavy-patterned substrate [5]. This results in a parabolic-like potential profile, as can be deduced by transmission electron microscopy (TEM) observations. The investigated wires have a thickness ranging between 30 and $45 \mathrm{~nm}$. The physical shapes and the potential profiles of the structures discussed in this work are shown in Fig. 1.

The patterning and etching technology can also be applied to fabricate zero-dimensional heterostructures

\section{RECTANGULAR QUAHTUM HIRES}
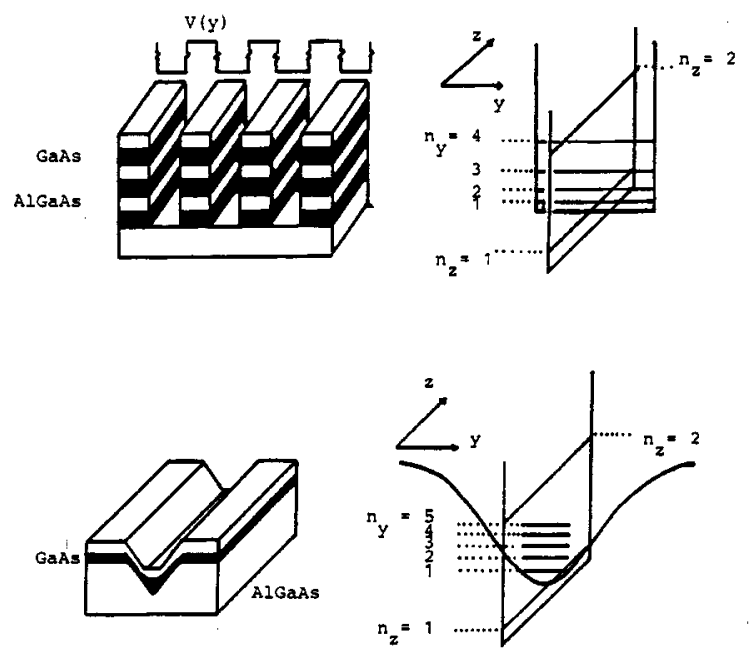

V-SHAPED QUANTUM WIRES

Fig. 1. Physical shape, confining potential and eigenstates of the quantum wire structures studied in this work, $n_{i}$ are the quantum numbers of the eigenstates resulting from the confinement along the i-direction. 
(quantum dots). Structures produced in this way reach a minimum size of the order of $25 \mathrm{~nm}$, and usually exhibit bad optical properties due to the damages induced by the processing [7]. An alternative way to obtain smaller OD systems $(2-10 \mathrm{~nm})$ is the precipitation of semiconductors in a transparent glass matrix, followed by heat treatment. In this work we present a few data on CdSe and $\mathrm{CdSeS}$ quantum dots of radius ranging between 2 and $6 \mathrm{~nm}$.

\section{Results and discussion}

\subsection{Search for quantum states}

The basic optical characterization of low-dimensional heterostructures becomes crucial when a clear description of the electronic states is needed. This also provides a feedback for the improvement of the fabrication technique and the necessary background for more advanced optical experiments and applications.

PL, PLE and PR experiments on GaAs/AlGaAs quantum wires of different sizes and shapes revealed several interesting phenomena. In narrow wires (below $40 \mathrm{~nm}$ ) a distinct evidence of optical transitions among true 1D states can be obtained. This is shown in Fig. 2 where we compare the photoreflectance and the photoluminescence spectra of a Vshaped $\mathrm{GaAs} / \mathrm{AlGaAs}$ quantum wire structure consisting of two bent quantum wells [see Fig. 2(a)]. The different regions of the heterostructure contribute to the optical spectra as

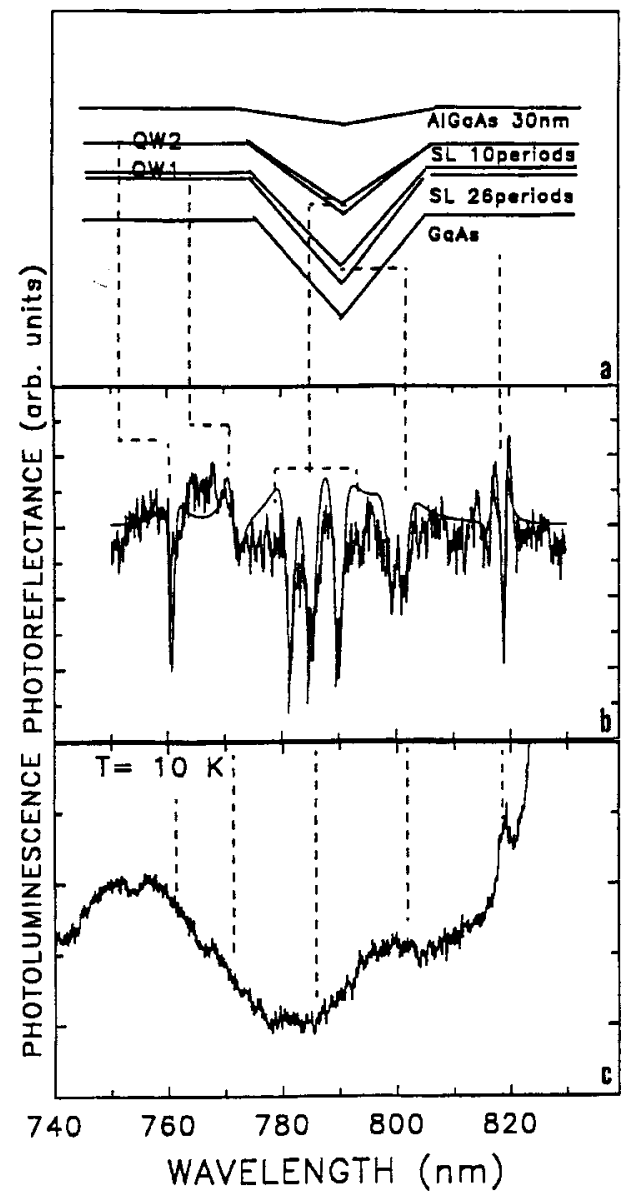

Fig. 2. Schematic cross-section (a), experimental and theoretical (smooth continuous line) photoreflectance spectra (b) and photoluminescence spectrum (c) of a V-shaped GaAs quantum wire structure. The measurements were performed at $10 \mathrm{~K}$. The vertical dashed lines attribute the spectral features to the different regions of the sample (see text). indicated in the figure. The top-flat part of the quantum wells between adjacent grooves gives an intense luminescence band [Fig. 2(c)] and sharp excitonic resonances [Fig. 2(b)] at energy positions consistent with the prediction of the envelope function calculations (the actual quantum well thickness was measured by TEM). The quantum wires form at the bottom of the V-shaped region (edge of the bent quantum well), where carriers are laterally confined by the progressive shrinkage of the quantum well on the sidewalls (as measured by TEM). The increasing confinement energies of carriers on both sidewalls is evaluated by the simple square well model and is then fitted by a parabolic-like potential $\left(1 / \cos h^{2}\right)$. The solution of the Schrödinger equation with this potential gives the set of eigenstates for the electrons at the bottom of the V-shaped well. For the sample shown in Fig. 2, an almost constant splitting of about $6-8 \mathrm{meV}$ among the quantized states is expected, according to the parabolic shape of the potential. In fact, three sharp resonances with almost constant energy splitting are observed from the quantum wire region [see Fig. 2(b)]. The constant energy separation and the spectral position of these features is consistent with the quantization energies of carriers confined by the two-dimensional confining potential of the heterostructure. It is interesting to note that in this sample the quantum wire luminescence is not intense [Fig. 2(c)], indicating that carriers photogenerated at the sample surface are not efficiently trapped in the wires. This is due to the periodicity of the grooves (about $450 \mathrm{~nm}$ ) which is longer than the carrier diffusion length, thus preventing carrier trapping at the bottom of the V-shaped region [6]. The luminescence from higher-index quantum states can be otherwise observed in samples with high density of wires, i.e. with an average quantum wire separation shorter than the electron mean free path. This is shown in Fig. 3 where we depict the temperature dependence of the PL of a GaAs

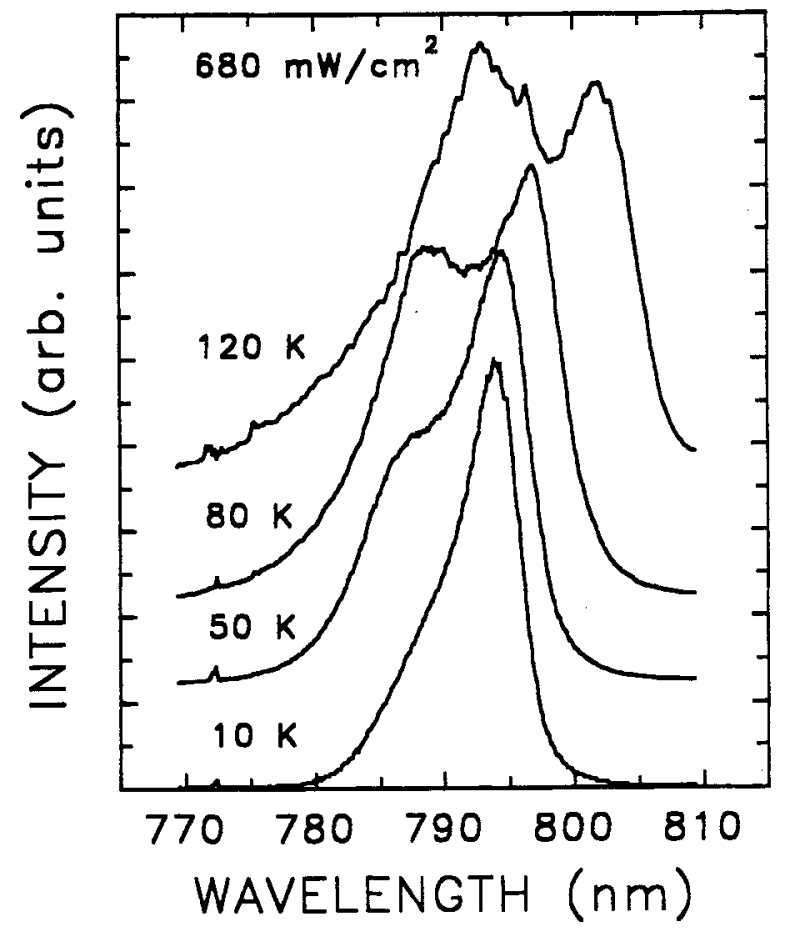

Fig. 3. Temperature dependent photoluminescence spectra of $\mathrm{V}$-shaped quantum wires with periodicity of $250 \mathrm{~nm}$. The thermal filling of the $n=2$ heavy-hole exciton is evident. 
quantum wire sample with a groove periodicity of about $250 \mathrm{~nm}$, and a structure similar to the one shown in Fig. 2. With increasing temperature, sharp PL peaks can be observed in the high energy tail of the fundamental $n=1$ transition. The energy splitting of about $17-19 \mathrm{meV}$ is consistent with the subband spacing calculated according to the well thicknesses measured by TEM. For this heterostructure an equivalent wire width of $25 \mathrm{~nm}$ is obtained.

Different results are found in rectangular quantum wire samples prepared by the patterning and etching technology, having a larger wire-width due to the technological limitations in the fabrication process. In these heterostructures the luminescence efficiency is usually found to reduce with reducing the wire width, as a consequence of the damage induced by the etching on the wire sidewalls [8]. In wire samples of intermediate thickness (around $60 \mathrm{~nm}$ ) we find that quantum size effects are differently pronounced under different experimental conditions: at low carrier densities, two-dimensional (2D) states are involved in the recombination processes, namely, quantized states of the excitonic center-of-mass motion. With increasing the carrier density, the exciton screening and the progressive filling of higher energy states allows the direct observation of optical transitions among true one-dimensional (1D) states. In fact in these samples the quantum wire width is significantly larger than the exciton Bohr radius. This is an intermediate size range in which excitons behave almost like two-dimensional particles and the lateral quantization in the wide quantum wire region (1D effect) mostly affects the exciton envelope function. This results in the quantization of the center-ofmass motion of the exciton as a whole, and no strict onedimensional confinement of the excitonic wave function occurs. Experimentally, the quantized center-of-mass (CM) states have been found to follow the expected quadratic dispersion in the exciton momentum with a discrete energy spectrum [9]. This results in the occurrence of sharp features in the PLE spectra related to the excited center of mass states with odd quantum numbers (allowed by parity conservation in the optical transitions). In Fig. 4 we compare the photoluminescence excitation spectra and the temperature dependence of the exciton center of mass states observed in PL. The PLE spectrum shows the $n=1, n=3$ and $n=5$ states at $1.5545 \mathrm{eV}, 1.5565 \mathrm{eV}$ and $1.559 \mathrm{eV}$, respectively, together with the light hole $(\mathrm{LH})$ peak at $1.564 \mathrm{eV}$. The light hole exciton does not show any additional confinement, probably due to the strong nonparabolicity which increases the light hole mass. The PLE features can be also observed in the temperature dependent PL spectra, exploiting the thermal filling of the excited center of mass states. The Lorentzian line-shape deconvolution of the PL spectra of Fig. 4 [10] allows to follow the energy shift of the CM states up to room temperature, as shown in the upper inset of Fig. 4. To support the exciton attribution, we also plot in the lower inset of Fig. 4 the temperature dependence of the integrated emission intensity of the excited center of mass states. As expected from thermodynamic considerations the emission intensity follows a Bose-Einstein population function, with activation energy given by the center of mass quantization energy (continuous lines). These excitonic features, can only be observed in relatively wide quantum wires, in which one-dimensional effects are not very pronounced, and quantum confinement basi-

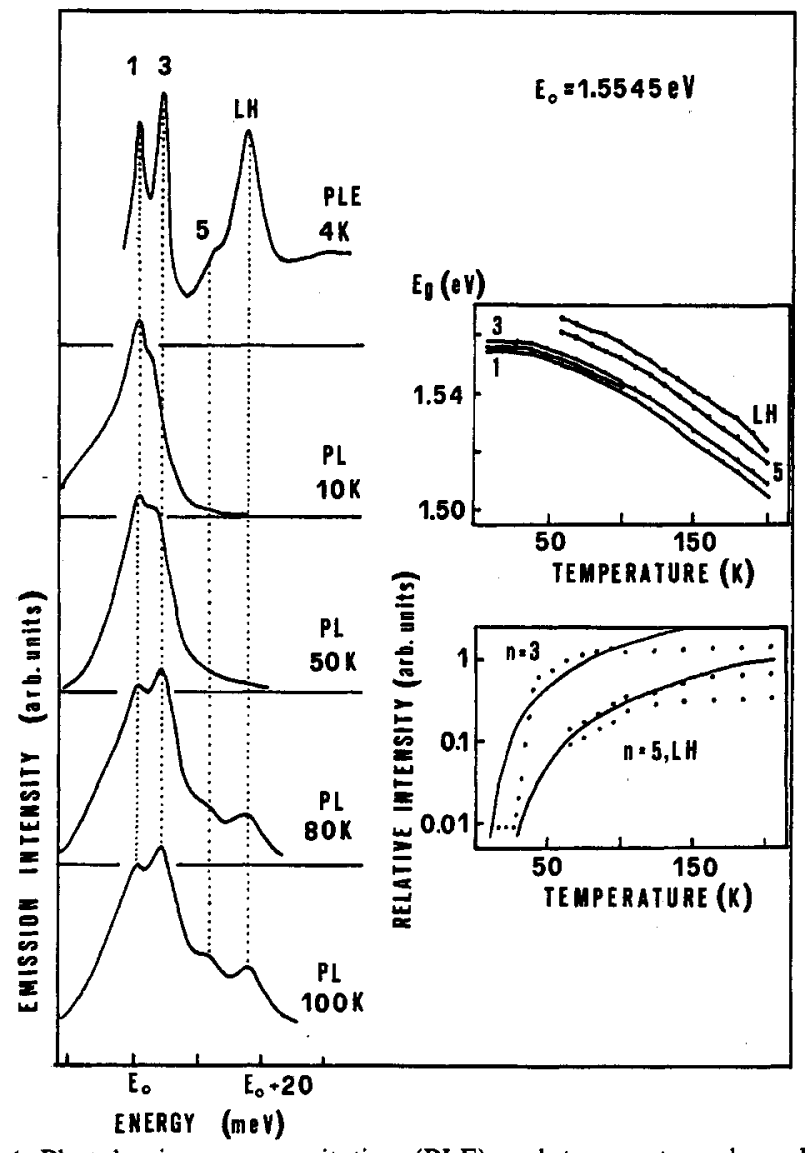

Fig. 4. Photoluminescence excitation (PLE) and temperature dependent photoluminescence (PL) spectra of rectangular GaAs/AlGaAs quantum wires of width $60 \mathrm{~nm}$. The excitation intensity is $0.5 \mathrm{~W} \mathrm{~cm}^{-2}$. The vertical dotted lines indicate the expected position of the allowed center of mass states $n=1,3,5$ and of the light-hole transition (LH). The PL spectra have been shifted to compare the transition splittings. In the upper inset we plot the temperature dependence of the peak energy of the excited center of mass states, deduced from the line-shape fitting of the PL spectra. The continuous lines are guides for the eye. The interrupted line belongs to the forbidden $n=2$ transition which cannot be included in the fit of the spectra above $90 \mathrm{~K}$. The lower inset compares the integrated emission intensity of the center of mass states (deduced from the fits) with the Bose-Einstein population functions (continuous lines), with activation energy equal to the quantization energy of the center of mass states.

cally affect the exciton envelope function as a whole, but not the individual carriers (weak confinement regime). Interband transitions among 1D states can be observed at higher excitation intensity, when the excitons are screened (see Section 3.2).

Before turning to the quantum confined electron-hole plasma properties, we briefly address the problem of the excitonic states in three-dimensionally confined systems (quantum dots). Besides the usual patterning and etching technology, nanometer quantum dots can be fabricated by precipitation and subsequent thermal treatment of semiconductors in a glass matrix. Although dots as small as $2 \mathrm{~nm}$ can be achieved in this way, the distribution of quantum dot size results polydispersed. This can be directly observed by TEM experiments, revealing different families of quantum dots with average radius $R_{i}$ and radius broadening $R_{i} \pm \Delta R_{i}$ around the average value [3]. A typical size histogram is shown in Fig. 5(a) for a CdSeS sample. The size polydispersion results in a complex absorption spectrum in which the excited states of the quantum dot families overlap in energy. Furthermore, a considerable spectral broadening is 

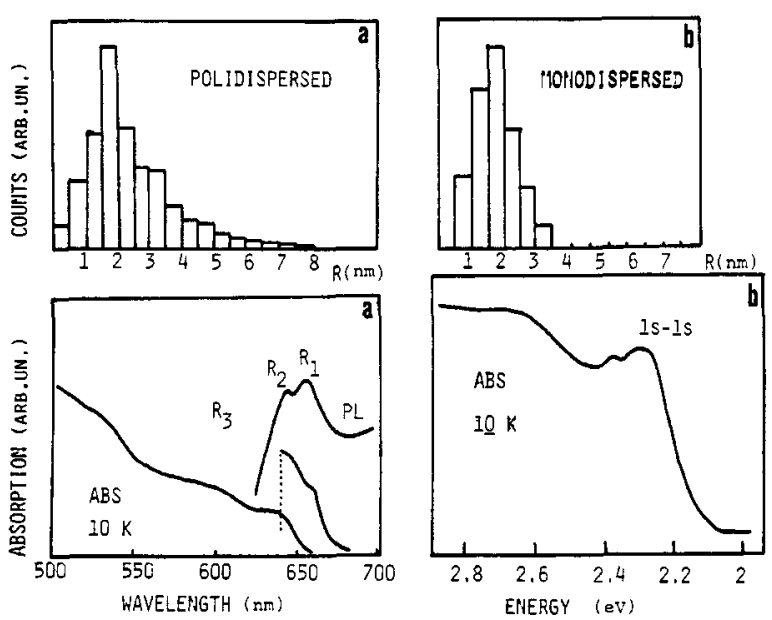

Fig. 5. Size histograms and absorption spectra of a polydispersed CdSeS quantum dot sample (a) and of a monodisperse CdSe quantum dot sample (b). $R_{i}$ indicate the absorption features related to the $1 s-1 s$ excitonic transition of dots with different size, as deduced by the Gaussian deconvolution of the size histograms.

observed due to the $\Delta R_{i}$ dispersion in the average radius of each dot family. In the absorption spectrum of Fig. 5(a) the transitions associated with different quantum dot families are labeled by $R_{i}$. The transition energies are calculated by the effective mass approximation, including Coulomb effects and exchange interaction [11]. This model can be successfully applied to these three-dimensionally confined systems of radius comparable to the exciton Bohr Radius in the crystal. Mass non-parabolicity, size dependent dielectric constant and strain effect should be included in order to correctly evaluate the energy gap of quantum dots below $2 \mathrm{~nm}$ [12]. The extreme size reduction of these systems makes quantum dots in glass matrix good candidates for photonic switching and non-linear modulators. Nevertheless, some effort has to be devoted to the fine control of the size dispersion. In Fig. 5(b) we show the size histogram of a monodispersed CdSe quantum dot sample, with main radius equal to $2 \mathrm{~nm}$ and radius dispersion of about $\pm 1 \mathrm{~nm}$. In this sample a distinct absorption feature can be observed, corresponding to the $1 \mathrm{~s}$ - $1 \mathrm{~s}$ exciton transition, and a shoulder due to the split-off band of CdSe around $2.6 \mathrm{eV}$. This kind of sample, though monodispersed, still presents a considerable broadening of the density of states (due to $\Delta R$ ) which has to be strongly reduced in order to exploit the advantageous delta-like density of states of quasi-zero dimensional systems.

\subsection{Many-body processes in quantum wires}

We now turn to the analysis of the PL spectra recorded under high excitation intensity (laser pulses of $25 \mathrm{ps}$ ) in the $60 \mathrm{~nm}$ quantum wires of Fig. 4. Under these conditions excitons are screened and free carriers are confined by an effective two-dimensional confining potential, thus filling the one-dimensional (1D) quantized states of the wire [13]. This is shown in Fig. 6, where we display the PL spectra at different delays after the exciting pulse (power density $2.5 \mathrm{MW} \mathrm{cm}^{-2}$ ). The spectra exhibit distinct intersubband transitions among 1D states. The energy positions of the peaks compare very well with the interband transition energies already calculated for this sample [13]. Spectra recorded at short delays exhibit a broad high energy tail due to the hot-carrier plasma formation. With increasing delays energy relaxation occurs and carriers thermalize at the lowest energy state. For delays as long as 600 ps only the fundamental $n_{\mathrm{y}}=1$ transition is observed. The observed photoluminescence lifetimes compare with those measured under identical excitation conditions in the quantum well from which the wires were fabricated. Information on the temporal evolution of the electron-hole plasma luminescence in these wires can be obtained by the calculation of the PL line shape, which reads:

$$
\begin{aligned}
I(\hbar \omega) \simeq & \sum_{j=1}^{N} \int \mathrm{d} E^{\prime} \int \mathrm{d} E_{\mathrm{e}} \int \mathrm{d} E_{\mathrm{h}} \delta\left(\hbar \omega-E_{\mathrm{e}}-E_{\mathrm{h}}-E_{\mathrm{g}}^{*}\right) \\
& \times f_{\mathrm{e}}\left(\varepsilon_{j \mathrm{e}}\right) f_{\mathrm{h}}\left(\varepsilon_{j \mathrm{~h}}\right) D\left(E^{\prime}-E_{\mathrm{g}}^{*}-E_{j \mathrm{e}}-E_{j \mathrm{~h}}\right) \\
& \times L_{\mathrm{e}}\left(E_{\mathrm{e}}, \varepsilon_{j \mathrm{e}}\left(E^{\prime}\right)\right) L_{\mathrm{h}}\left(E_{\mathrm{h}}, \varepsilon_{j \mathrm{~h}}\left(E^{\prime}\right)\right)
\end{aligned}
$$

where the summation is over $N$ occupied subbands, $E_{\mathrm{g}}^{*}$ is the renormalized band gap,

$\varepsilon_{j e, \mathrm{~h}}=\frac{m_{\mathrm{h}, \mathrm{e}}}{m_{\mathrm{e}}+m_{\mathrm{h}}} *\left(E^{\prime}-E_{\mathrm{g}}^{\times}-E_{j \mathrm{e}}-E_{j \mathrm{~h}}\right)+E_{j \mathrm{e}, \mathrm{h}}$

consistent with the assumption of momentum conservation in the recombination, and $D(E)$ is the one-dimensional density of states proportional to $E^{-1 / 2}$. In this model the quasiparticle broadening is included by means of a convolution of the single particle states with Lorentzian functions $L_{\mathrm{e}, \mathrm{h}}$ with constant damping parameters $\Gamma_{\mathrm{e}, \mathrm{h}}$.

The calculated line-shapes reproduce satisfactorily the experimental spectra. From the calculations we obtain the carrier density dependence and the temporal evolution of the band gap renormalization (BGR) shown in Figs 7(a) and 8 , respectively. The BGR ranges between 12 and $25 \mathrm{meV}$ in the density range $3 \times 10^{5} \mathrm{~cm}^{-1}<n<4 \times 10^{6} \mathrm{~cm}^{-1}$ and compares well with the theoretical calculations of Ref. [14]. We should mention that the available theoretical models do not include the population of higher energy subbands and the hole contribution to the exchange and correlation energies. The comparison is thus only indicative of some overall agreement between theory and experiment. Calculations for narrow wires with harmonic potential have also been reported, which cannot be directly compared to our data

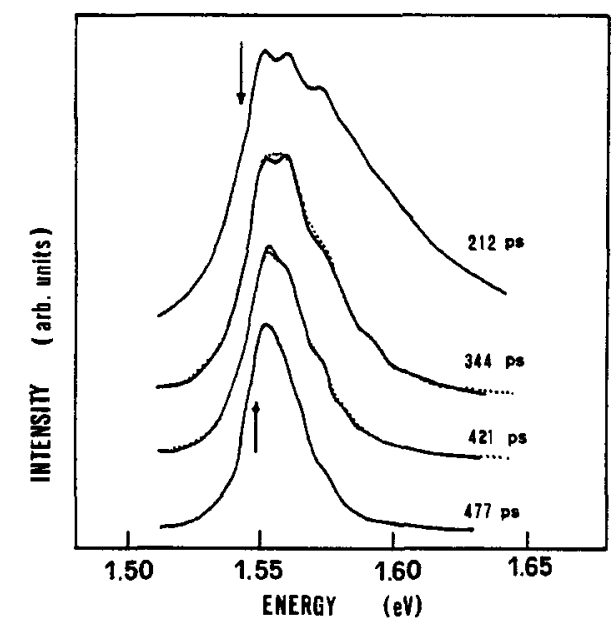

Fig. 6. Time resolved photoluminescence spectra of the GaAs quantum wires of Fig. 4, under excitation intensity of the order of $1 \mathrm{MW} \mathrm{cm}^{-2}$. The temperature is $4 \mathrm{~K}$. The dotted lines are the line shape fits calculated by eqs (1) and (2). 

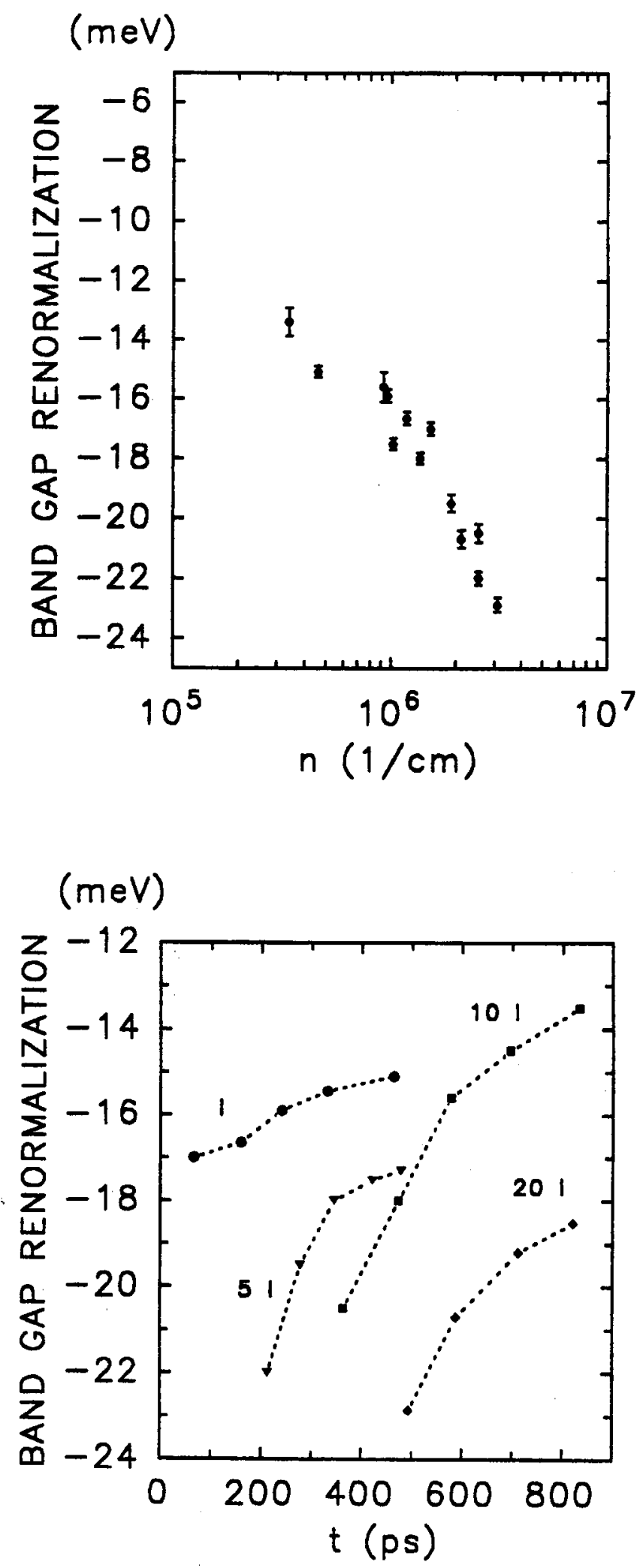

Fig. 7. Carrier density dependence (a) and temporal evolution (b) of the band gap renormalization in quantum wires, as extracted from the line shape fitting of the high-excitation-intensity time-resolved PL spectra recorded at different intensities $\left(I=0.5 \mathrm{MW} \mathrm{cm}^{-2}\right)$.

[15]. In addition, following the temporal evolution of the carrier density (which reduces due to recombination) the BGR is found to decrease in time. Due to the long carrier lifetime, the unperturbed value of the gap is recovered within about $1 \mathrm{~ns}$ in the present sample [Fig. 7(b)]. The temporal evolution of the carrier temperature can also be deduced by the line shape analysis. This is shown in Fig. 8

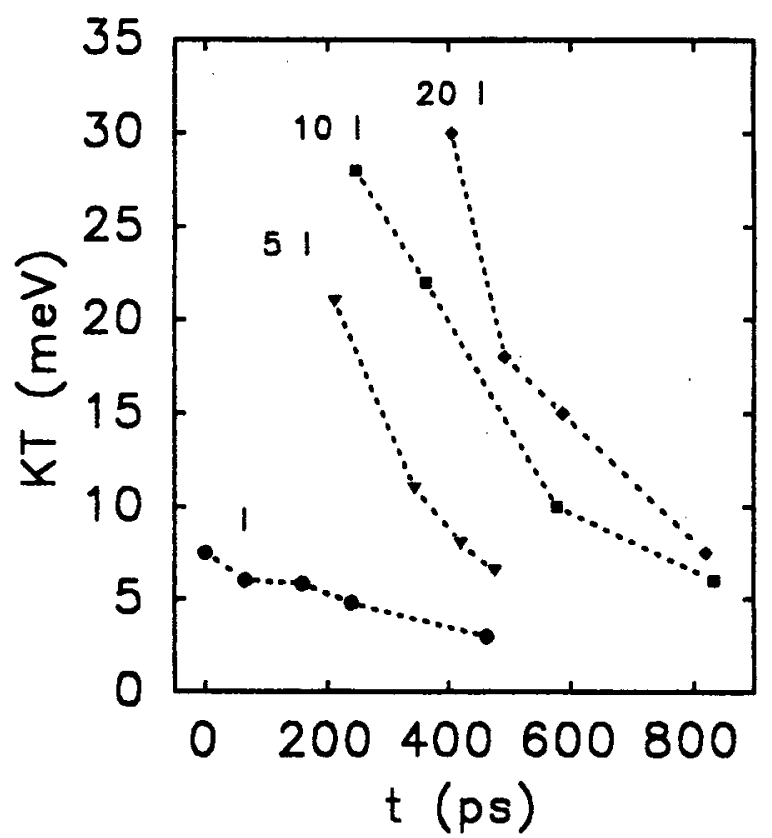

Fig. 8. Temporal evolution of the average carrier temperature (KT) obtained from the PL line shape fitting.

where we report the carrier cooling for different excitation intensities. At photogeneration rates of the order of $3 \times 10^{6} \mathrm{~cm}^{-1}$, the hot plasma is found to cool down in a time of the order of $1 \mathrm{~ns}$. Shorter times are obtained with reducing the carrier density. Also for the problem of the energy relaxation, so far no theories including multisubband populations have been reported, and the comparison with the available theoretical models is difficult [16]. However, the cooling rate obtained at the lowest excitation intensity compares reasonably well with the one calculated in Ref. [17] for quantum wire with a single subband occupied. More extended experimental work and more realistic theoretical calculations are necessary in order to demonstrate that the carrier cooling rate decreases in quantum confined systems.

\section{Conclusions}

In conclusion, we have shortly summarized the results of extensive spectroscopic studies of quantum wires and quantum dots. From the basic search of quantum states several information on the excitonic states and on the actual confinement mechanism of carriers can be deduced. True one-dimensional excitonic confinement or two-dimensional quantization of the exciton center of mass motion can be observed in GaAs wires in the size range $25-60 \mathrm{~nm}$. The effects connected to the different shapes of the confining potentials have been discussed in wire and dots of different composition and structure. Finally, the electron-hole plasma recombination has been discussed in order to get information on the band gap renormalization and on the temporal evolution of the carrier energy.

\section{Acknowledgements}

This work results from the collaboration between the GNEQP group of the University of Bari (Italy), The Max-Planck Institut für Festkörperforschung of Stuttgart (Germany), and the Ecole Politechnique Federale of Lausanne (Swiss). Special thanks are due to R. Rinaldi, H. Lage, G. C. LaRocca, D. Heitmann, U. Marti and F. K. Reinhart for their intense col- 
laboration to the research work. Part of the research work is sponsored by the ESPRIT project NANOPT.

\section{References}

1. For recent reviews on optical properties of quantum wells see: Schmitt-Rink, S., Miller, A. D. B. and Chemla, D. S., Adv. Phys. 38, 89 (1989); Cingolani, R. and Ploog, K., Adv. Phys. 40, 535 (1990).

2. Tapfer, L., LaRocca, G. C., Lage, H., Cingolani, R., Grambow, P., Fisher, A., Heitmann, D. and Ploog, K., Surf. Sci. 267, 227 (1992).

3. Cingolani, R., Moro, C., Manno, D., Striccoli, M., DeBlasi, C., Righini, G. C. and Ferrara, M., J. Appl. Phys. 70, 6989 (1991).

4. Cingolani, R., Lepore, M., Tommasi, R., Catalano, I. M., Lage, H., Heitmann, D., Ploog, K., Shimizu, A., Sakaki, H. and Ogawa, T., Phys. Rev. Lett. 69, 1276 (1992).

5. Kapon, E., Hwang, D. M. and Bhat, R., Phys. Rev. Lett. 63, 430 (1989).

6. Walther, M., Kapon, E., Hwang, D. M., Kolas, E. and Nunes, L., Phys. Rev. B45, 6333 (1992).
7. Clausen, E. M., Craighead, H. G., Worlock, J. M., Harbison, J. P., Schiavone, L. M., Florez, L. and Van der Gaag, B., Appl. Phys. Lett. 55, 1427 (1989).

8. Maile, B. E., Forchel, A., German, R. and Grützmacher, D., Appl. Phys. Lett. 54, 1552 (1989).

9. Lage, H., Heitmann, D., Cingolani, R., Grambow, P. and Ploog, K., Phys. Rev. B44, 6550 (1991).

10. Rinaldi, R., Cingolani, R., Ferrara, M., Lage, H., Heitmann, D. and Ploog, K., Phys. Rev. 47, (1993), in press.

11. Kayanuma, Y., Phys. Rev. B38, 9797 (1988).

12. For precise tight-binding calculations see: Lippens, P. E. and Lannoo, M., Phys. Rev. B39, 10935 (1985).

13. Cingolani, R., Lage, H., Tapfer, L., Kalt, H., Heitmann, D. and Ploog, K., Phys. Rev. Lett. 67, 891 (1991).

14. Ben Yu-Kuang and Sarma, S. Das., Phys. Rev. B68, 1750 (1992).

15. Benner, S. and Haug, H., Europhys. Lett. 16, 579 (1991).

16. Rota, L., Rossi, F., Goodnick, S. M., Lugli, P., Molinari, E. and Porod, W., Phys. Rev. B47, 1632 (1993).

17. Campos, V. B. and Sarma, S. Das., Phys. Rev. B45, 3898 (1992). 